\title{
Sex disparities in dietary intake across the lifespan: the case of Lebanon
}

Lara Nasreddine $^{1 \dagger}$, Marie Claire Chamieh ${ }^{1 \dagger}$, Jennifer Ayoub ${ }^{1}$, Nahla Hwalla$^{1}$, Abla-Mehio Sibai $^{2}$ and Farah Naja ${ }^{1 *}$

\begin{abstract}
Background: Little is known about sex-based dietary differences in middle-income countries, particularly those undergoing the nutrition transition. This study aims at examining sex disparities in energy and macronutrients' intakes, food consumption patterns, and micronutrients' adequacy in Lebanon, while adopting a life course approach.
\end{abstract}

Methods: Data were derived from a national cross-sectional survey conducted in Lebanon in 2008/2009. The study sample consisted of 3636 subjects: 956 children and adolescents aged 6-19.9 years; 2239 adults aged 20-59.9 years and 441 older adults aged above 60 years. At the households, trained nutritionists conducted face-to-face interviews with participants to complete a sociodemographic questionnaire and one 24-h diet recall. Food items were categorized into 25 food groups. The Nutritionist Pro software was used for the analysis of dietary intake data and the estimation of energy, macronutrients', and micronutrients' intakes.

Results: In all age groups, males had significantly higher energy intakes, while females had significantly higher fiber intakes. In addition, in adolescents aged 12-19.9 years, females had higher fat intakes as compared to males (37.02 \pm $0.6 \%$ vs $35.03 \pm 0.61 \%$ ), and in adults aged $20-59.9$ years, females had significantly higher total fat $(37.73 \pm 0.33 \%$ vs $36.45 \pm 0.38 \%)$ and saturated fat intakes (11.24 $\pm 0.15 \%$ vs $10.45 \pm 0.18 \%)$. These differences in macronutrient intakes were not observed in younger children nor in older adults. Sex-based differences in food groups' intakes were also observed: men and boys had significantly higher intakes of red and processed meat, bread, fast food, soft drinks, and alcohol, while girls and women had higher intakes of fruits, vegetables, milk, and sweets. In all age groups, females had lower micronutrient intakes compared to males, including calcium, iron, and zinc.

Conclusions: This study identified sex-specific priorities that ought to be tackled by context-specific interventions to promote healthier diets in Lebanon. The fact that sex-based differences in nutrient intakes and food consumption patterns were the most noticeable in the adolescent and adult years, hence women's reproductive years call for concerted efforts to improve nutrition for women and girls as this would lay the foundation not only for their future education, productivity, and economic empowerment, but also for the health of future generations.

Keywords: Sex-based differences, Dietary intakes, Food consumption patterns, Food groups, Macronutrients, Micronutrients, Nutrition, Lebanon

\footnotetext{
* Correspondence: fn14@aub.edu.lb

${ }^{\dagger}$ Lara Nasreddine and Marie Claire Chamieh contributed equally to this work. 'Department of Nutrition and Food Science, Faculty of Agricultural and Food Sciences, American University of Beirut, Beirut, Lebanon

Full list of author information is available at the end of the article
}

C C The Author(s). 2020 Open Access This article is licensed under a Creative Commons Attribution 4.0 International License, which permits use, sharing, adaptation, distribution and reproduction in any medium or format, as long as you give appropriate credit to the original author(s) and the source, provide a link to the Creative Commons licence, and indicate if changes were made. The images or other third party material in this article are included in the article's Creative Commons licence, unless indicated otherwise in a credit line to the material. If material is not included in the article's Creative Commons licence and your intended use is not permitted by statutory regulation or exceeds the permitted use, you will need to obtain permission directly from the copyright holder. To view a copy of this licence, visit http://creativecommons.org/licenses/by/4.0/. The Creative Commons Public Domain Dedication waiver (http://creativecommons.org/publicdomain/zero/1.0/) applies to the data made available in this article, unless otherwise stated in a credit line to the data. 


\section{Introduction}

Goal 5 of the United Nations' Sustainable Development Goals (SDGs) aims to "achieve gender equality and empower all women and girls" around the world. While women and girls often have increased nutritional needs during the lifecycle, social norms in many parts of the world frequently lead to gender inequalities in nutrition, which tend to disfavor females [1]. Worldwide, women and girls continue to be twice as likely to suffer from all forms of malnutrition compared to their male counterparts, highlighting the need for a better understanding of sex disparities in food consumption and dietary intakes as a determinant of health [1]. In this manuscript, and based on the recommendations of the Institute of Medicine, the term "sex" is used as a biological classification determined by sex chromosomes, while the term gender is used when referring to behavioral, cultural, or psychological traits typically associated with one sex $[2,3]$.

Evidence stemming from different parts of the world depicts conflicting pictures of sex-based discrepancies in diet and nutrition [4-7]. Studies conducted in developing, low income countries, showed that women and girls have lower intakes of nutrientdense foods such as meats, eggs, milk, pulses, fruits, and vegetables and a higher risk for micronutrients and chronic energy deficiencies compared to males [8]. However, in Western developed countries, studies have shown that females tend to make healthier food choices and consume higher amounts of fruits, vegetables, and dietary fiber compared to males [9]. Little is known about sex-based dietary differences in middle-income countries, particularly those undergoing the nutrition transition, with its characteristic shifts in diet and lifestyle [5]. This may be particularly true for the Eastern Mediterranean Region (EMR), which has witnessed rapid changes in food consumption habits and dietary practices over the past decades. The region is also characterized by a double burden of malnutrition with strong female vs. male disparities: Women and girls in the EMR have a higher burden of micronutrient deficiencies while also being approximately three times more prone to obesity compared to males [10-12]. Apart from sex-based physiological and biological differences, a variety of external factors may explain these gender disparities, including the non-egalitarian household and social roles in the region [13-16].

Lebanon, a small country of the EMR, has made substantial achievements with regard to gender equality and empowerment $[17,18]$ over the past 10 years. However, similar to other countries of the region, the country still harbors a set of traditions and social norms that may result in unequal gender roles both within the household and in the society [5]. These include unequal division of household chores, lower expectations for women regarding education and/or professional achievements, and gender-based constraints on physically active leisure activities in addition to social legislations disfavoring women $[5,19]$. It is important to understand whether these non-egalitarian social roles in Lebanon are also associated with disparities in diet and food consumption, particularly that sex-based disparities in the burden of micronutrient deficiencies' and obesity prevalence were previously reported $[12,20]$. Interestingly, previous studies in Lebanon showed that sex differentials in obesity prevalence were not static, but were rather dynamic across the lifespan. For instance, while obesity rates did not differ between boys and girls in young children, significant sex-based disparities were noted in the adolescent age group, with the prevalence of obesity being significantly higher in boys compared to girls [21]. Among adults, men showed higher prevalence rates at the younger age groups (20-49 years), while women showed higher prevalence rates in older age groups (50 years and above). These differentials were most apparent in obesity class II and class III with women being significantly more likely than men to show this type of excessive adiposity [20].

Acknowledging the need for a better understanding of gender-based differences in dietary intakes, this study aims at examining sex-based disparities in energy and macronutrients' intakes, food consumption patterns, and micronutrients' adequacy in Lebanon, while adopting a life course approach. The characterization of sex-based disparities in dietary intakes would be useful to set priorities and inform the development and implementation of comprehensive interventions aimed at reducing inequalities in food and nutrient intakes across the lifespan and subsequently enhancing the population's nutritional status and health profile [4].

\section{Methods}

The data for the present study were derived from the national cross-sectional food consumption survey conducted in Lebanon between May 2008 and August 2009. Details about the design and protocol of this survey are found elsewhere [22, 23]. In brief, the survey was based on the sampling frame provided by the Ministry of Social Affairs/Central Administration of Statistics in collaboration with United Nations Development Programme (UNDP). Efforts were exerted to recruit a nationally representative sample with age, sex, and district distributions proportionate to that of the Lebanese population $[24,25]$. The study sample consisted of randomly selected households, based on stratified cluster sampling: the strata were the 
Lebanese governorates, the clusters were selected further at the level of districts, urban, and rural areas, and the housing units constituted the primary sampling units. One adult from each household and one child/adolescent from every other household were selected from the household roster, excluding pregnant and lactating women and subjects with mental disabilities. The study sample consisted of 3636 subjects (956 children and adolescents aged 6-19.9 years; 2239 adults aged 20-59.9 years and 441 older adults aged above 60 years) (response rate: $89.3 \%$ ). The design and conduct of the survey were approved by the Institutional Review Board of the American University of Beirut, and informed consents from adults/parents and informed assents from children and adolescents were obtained prior to enrollment in the study.

At the households, trained nutritionists conducted face-to-face interviews with participants to complete a sociodemographic questionnaire and one 24-h diet recall (24-HR). The questionnaire covered information related to sex (male, female), age (in years), and governorate of current residence (Beirut, Mount Lebanon, North, South, Bekaa, and Nabatieh). For children and adolescents aged between 6 and 19.9 years, questions about mother's and father's educational levels (primary education or less, up to high school education, and university degree or higher) were included in the questionnaire. For adults aged 20 years and above, sociodemographic information collected were related to crowding index, marital status (single, married, or divorced/separated/ widowed), educational level (primary education or less, up to high school education, and university degree or higher), and employment status (employed, not employed, housewife, or student). In this survey, crowding index was used as a proxy for socioeconomic status. This index is calculated as the ratio of the number of people living in the household over the number of rooms, excluding the kitchen and bathrooms. Several epidemiological studies have associated a high household crowding index with low socioeconomic status [26, 27].

Dietary intake was examined using the 24-HRs referring to dietary intake consumption during the previous $24 \mathrm{~h}$. The 24-HRs in this study were carried out using the Multiple Pass Food Recall 5-step approach, developed by the United States Department of Agriculture (USDA) [28]. This approach has consistently showed attenuation in the 24-HRs' limitations [29]. The five steps followed included 1) quick food list recall, 2) forgotten food list probe, 3) time and occasion at which foods were consumed, 4) detailed overall cycle, and 5) final probe review of the foods consumed. For children up to 12 years of age, the mother was considered as proxy. In case the responsibility for feeding of the child was shared with another caregiver (father, helper, or other), the mother was encouraged to consult with the caregiver to obtain additional/ complementary details about the dietary intake of the child. For adolescents (aged between 12 and 19.9 years), participants were interviewed directly to complete the 24-HRs and caregivers were consulted if further details were needed regarding ingredients, methods of preparation, and portion sizes. The latter was determined using quantification tools [30] and which included standard measuring cups and spoons, household measures, as well as food photos and models of single servings of commonly consumed food items. The nutritionists who were collecting the dietary intake data were specifically trained to maintain a neutral attitude and avoid leading questions.

The Nutritionist Pro software (version 5.1.0, 2014, First Data Bank, Nutritionist Pro, Axxya Systems, San Bruno, CA) was used for the analysis of dietary intake data and the estimation of energy, macronutrients', and micronutrients' intakes. For composite and mixed dishes, standardized recipes were added to the Nutritionist Pro Software using single food items. Within the Nutritionist Pro, the USDA database was selected for analysis (SR 24, published September 2011). Food composition of specific Lebanese foods (not included in the Nutritionist Pro software database) was obtained from local food composition tables [31]. In addition to energy, macronutrients, and micronutrients, the dietary intake data was also used to obtain information about food groups intakes. Food items were categorized into the following 25 food groups: bread, cereals, legumes, starchy vegetables, vegetables, chips and salty crackers, nuts and seeds, milk, milk derivatives, sweetened milk, red meat, processed meat, poultry, fish, eggs, fruits, fresh fruit juices, sweets, added sugars, sugar sweetened beverages, hot beverages (coffee, tea), alcoholic beverages, added fats and oils, fast food, and miscellaneous. Food items included within each of the aforementioned food groups are outlined in Appendix A.

\section{Statistical analyses}

The data for this study were presented and compared between sexes among the various age groups (6-19.9 years, 20-59.9 years, and $\geq 60$ years). In addition, data for adolescents (aged 12-19.9 years) were examined and presented separately in Appendix B. Sociodemographic and dietary intake variables were described by means \pm standard error (SE) and proportions and were compared between males and females using $t$ test and chi square test, for continuous and categorical variables, respectively. Macronutrients' and food groups' intakes were expressed as percent of total en-

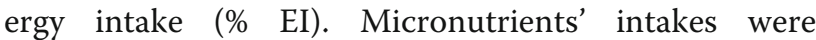


presented as means $\pm \mathrm{SE}$, as well as percent of the population meeting 2/3rd of the Recommended Daily Allowance (RDA). The means \pm SE of dietary intakes were adjusted for differences in sociodemographic variables among males and females, using Analysis of Covariance (ANCOVA). In the latter, the sociodemographic covariates included were those that showed statistical difference between males and females. The Statistical Package for Social Sciences 22.0 (SPSS for Windows, 2013, Chicago: SPSS Inc.) was used for the statistical analyses. All statistical analyses were twotailed and a $p$-value $<0.05$ was considered statistically significant.

\section{Results}

Participants with missing dietary intake data were not included in the study $(n=149)$. In addition, a total of 93 subjects were excluded as outliers, based on total energy intake and using the interquartile range method [32]. Hence the number of participants included in this study was $n=3394$. Sociodemographic characteristics of the study population by age and sex are presented in Table 1. Among children and adolescents, the proportions of participants aged 6-11.9 and $12-19.9$ years were 42.8 and $57.2 \%$ respectively. As for governorate, the highest proportions of participants came from Mount Lebanon (37.1\%) and the North (23.5\%), with the lowest proportions being from Beirut (6.7\%) and Nabatieh (5.4\%). Most of the children and adolescents in this age group belonged to households with a crowding index $\geq 1$ (80.9\%). The distributions of the mother and father's education levels were similar, whereby the majority had 'up to a high school' level (father's education: 53.4\%, mother's education: 59.3\%) (Table 1). A comparison of sociodemographic characteristics between males and females in this age group showed a significant difference in the distribution across governorates $(p=0.003)$ (Table 1 ). Among adults aged 20 years and older, the study population consisted of $83.2 \%$ adults aged between 20 and 59.9 years and $16.8 \%$ older than 60 years. Over $40 \%$ of subjects were from Mount Lebanon (43.7\%) and $62.2 \%$ had a crowding index $\geq 1$. Sixty percent of participants $(60.5 \%)$ were married, $31.2 \%$ single, and $8.3 \%$ divorced, separated, or widowed. As for the education level, almost one in two participants (49.7\%) had 'up to a high school' level and $29.1 \%$ had a university degree or higher. Similarly, one in two participants (49.6\%) reported being 'employed', while 10.4\% were 'not employed', 33\% were housewives, and 7\% were students (Table 1). The comparison between males and females among adults in the study population showed significant differences related to all variables, except for education where the distributions of males and females across the three levels of education were not statistically different. More specifically, the proportion of older adults ( $\geq 60$ years) was higher among males than females $(18.8 \%$ vs $15.1 \%, p=$ 0.011). As for governorates, Beirut and Mount Lebanon had greater proportions of males, while Bekaa and Nabatieh had greater proportions of females. The proportion of females with a crowding index $\geq 1$ was higher than males $(64.4 \%$ vs $59.6 \%, p=$ 0.013). Furthermore, a higher proportion of females was divorced, separated, or widowed as compared to males $(12.2 \%$ vs $3.5 \%, \mathrm{p}<0.001)$. As for employment status, $75.4 \%$ of males vs $28.5 \%$ of females reported being 'employed' ( $\mathrm{p}<0.001)$ (Table 1 ).

Energy and macronutrients' intakes (expressed as percentage of total energy) of the study population are presented in Table 2. Among children and adolescents, energy intake was $1999.81 \pm 27.17 \mathrm{kcal}$ and the percentage contributions of macronutrients to energy were as follows: Carbohydrates: $51.57 \pm 0.34 \%$; proteins: $13.24 \pm 0.13 \%$; fat: $36.11 \pm 0.31 \%$. Saturated fat and total sugars made up $10.86 \pm 0.17 \%$ and $12.01 \pm$ $0.27 \%$ respectively. Fiber intake was estimated at $7.47 \pm 0.13 \mathrm{~g} / 1000 \mathrm{kcal}$. In this age group, comparison between males and females showed that males had a higher energy intake, while females had higher fiber intakes (Table 2). When adolescents (12-19.9 years) were examined separately, fat intake was shown to be significantly different among sexes, in addition to energy and fiber intakes. More specifically, females had higher fat intakes as compared to males $(37.02 \pm 0.6 \%$ vs $35.03 \pm 0.61 \%$ ) (Appendix B-Table 1). Among adults between 20 and 59.9 years, the contributions of macronutrients to energy were as follows: $48.8 \pm$ $0.25 \%$ for carbohydrates, $14.66 \pm 0.11 \%$ for proteins, and $37.09 \pm 0.23 \%$ for fat. In this age group, while males had significantly higher energy intakes, females had higher fat, saturated fat, and fiber intakes. Among older adults, the contributions of macronutrients to total energy intake were $49.48 \pm 0.58 \%$ for carbohydrates, $14.88 \pm 0.25 \%$ for proteins, and $36.27 \pm 0.52 \%$ for fat. In this age group, the comparison of energy and macronutrients' intakes between males and females showed similar results as those for children and adolescents, whereby males had higher energy intakes while females had higher fiber intakes (Table 2).

The contributions of the various food groups to energy intakes by sex among different age groups are presented in Table 3. Among children and adolescents, the percent contributions of the following food groups were higher in males as compared to females: red meat $(5.53 \pm 0.47 \%$ vs $3.96 \pm 0.47 \%)$, sugar sweetened beverages $(7.16 \pm 0.34 \%$ vs $5.73 \pm 0.34 \%)$, and fast food $(3.37 \pm 0.42 \%$ vs $1.65 \pm 0.42 \%)$. On the other 
Table 1 Sociodemographic characteristics by gender of the total study population $(n=3394)$

\begin{tabular}{|c|c|c|c|c|}
\hline \multicolumn{5}{|l|}{ Children and Adolescents } \\
\hline & $\begin{array}{l}\text { Total } \\
(n=865)\end{array}$ & $\begin{array}{l}\text { Males } \\
(n=437)\end{array}$ & $\begin{array}{l}\text { Females } \\
(n=428)\end{array}$ & $p$-value \\
\hline \multicolumn{5}{|l|}{ Age (years) } \\
\hline $6-11.9$ & $370(42.8)$ & $193(44.2)$ & $177(41.4)$ & \multirow[t]{2}{*}{0.404} \\
\hline $12-19.9$ & $495(57.2)$ & $244(55.8)$ & $251(58.6)$ & \\
\hline \multicolumn{5}{|l|}{ Governorate } \\
\hline Beirut & $56(6.7)$ & $34(8.1)$ & $22(5.2)$ & \multirow[t]{6}{*}{0.003} \\
\hline Mount Lebanon & $311(37.1)$ & $178(42.6)$ & $133(31.6)$ & \\
\hline North & $197(23.5)$ & $92(22.0)$ & $105(24.9)$ & \\
\hline South & $117(13.9)$ & $47(11.2)$ & 70 (16.6) & \\
\hline Bekaa & $113(13.5)$ & $48(11.5)$ & $65(15.4)$ & \\
\hline Nabatieh & $45(5.4)$ & $19(4.5)$ & $26(6.2)$ & \\
\hline \multicolumn{5}{|l|}{ Crowding index } \\
\hline$<1$ Person/Room & $165(19.1)$ & $89(20.4)$ & $76(17.8)$ & \multirow[t]{2}{*}{0.337} \\
\hline$\geq 1$ Person/Room & $699(80.9)$ & $348(79.6)$ & $351(82.2)$ & \\
\hline \multicolumn{5}{|l|}{ Father's education level } \\
\hline Primary or lower & $236(30.8)$ & $115(29.9)$ & $121(31.7)$ & \multirow[t]{3}{*}{0.467} \\
\hline Up to high school & $409(53.4)$ & $213(55.5)$ & $196(51.3)$ & \\
\hline University or higher & $121(15.8)$ & $56(14.6)$ & $65(17.0)$ & \\
\hline \multicolumn{5}{|l|}{ Mother's education level } \\
\hline Primary or lower & $192(24.3)$ & $101(25.8)$ & $91(22.9)$ & \multirow[t]{3}{*}{0.405} \\
\hline Up to high school & $468(59.3)$ & $233(59.4)$ & $235(59.2)$ & \\
\hline University or higher & $129(16.3)$ & $58(14.8)$ & $71(17.9)$ & \\
\hline \multicolumn{5}{|l|}{ Adults } \\
\hline & $\begin{array}{l}\text { Total } \\
(n=2529)\end{array}$ & $\begin{array}{l}\text { Males } \\
(n=1141)\end{array}$ & $\begin{array}{l}\text { Females } \\
(n=1388)\end{array}$ & $p$-value* \\
\hline \multicolumn{5}{|l|}{ Age (years) } \\
\hline $20-59.9$ & $2105(83.2)$ & $926(81.2)$ & $1179(84.9)$ & \multirow[t]{2}{*}{0.011} \\
\hline$\geq 60$ & $424(16.8)$ & 215 (18.8) & $209(15.1)$ & \\
\hline \multicolumn{5}{|l|}{ Governorate } \\
\hline Beirut & $281(11.1)$ & $153(13.4)$ & $128(9.2)$ & \multirow[t]{6}{*}{$<0.001$} \\
\hline Mount Lebanon & $1104(43.7)$ & $523(45.8)$ & $581(41.9)$ & \\
\hline North & $420(16.6)$ & $188(16.5)$ & $232(16.7)$ & \\
\hline South & $311(12.3)$ & $130(11.4)$ & $181(13.0)$ & \\
\hline Bekaa & $296(11.7)$ & $106(9.3)$ & $190(13.7)$ & \\
\hline Nabatieh & $117(4.6)$ & $41(3.6)$ & $76(5.5)$ & \\
\hline \multicolumn{5}{|l|}{ Crowding index } \\
\hline$<1$ Person/Room & $951(37.8)$ & $459(40.4)$ & $492(35.6)$ & \multirow[t]{2}{*}{0.013} \\
\hline$\geq 1$ Person/Room & $1567(62.2)$ & $677(59.6)$ & $890(64.4)$ & \\
\hline \multicolumn{5}{|l|}{ Marital status } \\
\hline Single & $788(31.2)$ & $429(37.6)$ & $359(25.9)$ & \multirow[t]{3}{*}{$<0.001$} \\
\hline Married & $1530(60.5)$ & $671(58.9)$ & $859(61.9)$ & \\
\hline Divorced, separated, or widowed & $209(8.3)$ & $40(3.5)$ & $169(12.2)$ & \\
\hline \multicolumn{5}{|l|}{ Education level } \\
\hline Primary or lower & $534(21.1)$ & $253(22.2)$ & $281(20.2)$ & 0.488 \\
\hline
\end{tabular}


Table 1 Sociodemographic characteristics by gender of the total study population $(n=3394)$ (Continued)

\begin{tabular}{llll}
\hline Children and Adolescents & & & \\
\hline Up to high school & $1258(49.7)$ & $562(49.3)$ & $696(50.1)$ \\
$\quad$ University or higher & $737(29.1)$ & $326(28.6)$ & $411(29.6)$ \\
Employment status & & & $395(28.5)$ \\
Employed & $1254(49.6)$ & $859(75.4)$ & $59(4.3)$ \\
Not employed* & $262(10.4)$ & $203(17.8)$ & $834(60.1)$ \\
Housewife & $835(33.0)$ & $1(0.1)$ & $100(7.2)$ \\
Student & $177(7.0)$ & $77(6.8)$ & $<0.001$ \\
\hline
\end{tabular}

Values in this table represent $\mathrm{n}(\%)$

" $p$-value derived from chi square test

${ }^{* *}$ Not employed included not working, does not want to work, looking for work, or retired

Bolded numbers are significant at $p<0.05$

hand, females had higher contributions of whole fruits to their total energy intake than males $(4.33 \pm 0.28 \%$ vs $2.71 \pm 0.28 \%)$. Among adolescents (12-19.9 years), the contributions of bread, processed meat, and fast food to total energy intake were higher among males than females, while those for nuts and seeds, whole fruits, and sweets were higher among females (Appendix B-Table 2). Among adults (20-59.9 years), further differences in food groups' contributions to total energy intakes were observed, whereby males had greater intakes of bread, red meat, sugar sweetened beverages, alcoholic beverages, and fast food, while females had higher intakes of vegetables, milk and milk derivatives, whole fruits, sweets, and hot beverages. Among older adults ( $\geq 60$ years), fewer differences in food groups' intakes were observed as compared to younger age groups, with only vegetables intakes being higher among females (Table 3).

Micronutrients' intakes in the study population, in terms of means $\pm \mathrm{SE}$ and proportions of participants below 2/3rd of the RDA, are presented in Table 4 . Among children and adolescents, the proportions of participants below $2 / 3$ rd of the RDA were $77.3 \%$ for calcium, $36.4 \%$ for iron, $26.6 \%$ for zinc, $55.3 \%$ for vitamin A, $26.2 \%$ for vitamin C, and $35.7 \%$ for vitamin B12. In this age group, except for vitamins $\mathrm{A}$ and $\mathrm{C}$, the proportions of participants falling below $2 / 3$ rd of the RDA were significantly higher among females than males for the micronutrients considered in this study. Similar results were obtained when the data for adolescents (aged 1219.9 years) were analyzed separately (Appendix B-Table $3)$. For adults (20-59.9 years), females had significantly lower mean intakes of all micronutrients, and consequently the proportions of females consuming less than 2/3rd of the RDA were higher than males, except for vitamin $\mathrm{A}$ and vitamin $\mathrm{C}$ where no statistical difference was noted. Among older adults (age $\geq 60$ years), the proportions consuming below 2/3rd of the RDA for calcium, iron, zinc, vitamin A, vitamin $\mathrm{C}$, and vitamin B12 were $79.2,29,42,67.5,45.1$, and $50 \%$ respectively. The proportions of females consuming less than $2 / 3 \mathrm{rd}$ of the RDA was significantly higher than those of males for calcium and iron (Table 4).

\section{Discussion}

Our study adopted a life course approach to investigate sex-based differentials in dietary intakes and food consumption patterns in Lebanon, a country where inequalities in the prevalence of obesity and micronutrient deficiencies were reported between sexes. Two consistent observations across the lifespan were the higher intake of energy in males compared to females and the higher intake of dietary fiber in females compared to males. Sex-based differences in macronutrients' intakes (\% EI) and food consumption patterns were also observed. Females had a higher intake of total fat and a higher consumption of fruits, vegetables, milk, and sweets (\% EI). On the other hand, males were found to have higher consumptions of red and processed meat, bread, fast food, soft drinks, and alcohol. Another consistent observation was the lower micronutrient intakes in females compared to males, across the lifespan.

The observed higher intakes of energy in males compared to females from all age groups are consistent with the well-established sex-based disparities in energy intakes, which reflect differences in physiological and metabolic factors $[4,33]$. Dietary fiber intakes ranged between 6.9 and $11.6 \mathrm{~g} / 1000 \mathrm{kcal}$, highlighting a gap between the population's current intake levels and nutritional recommendations $(14 \mathrm{~g} / 1000 \mathrm{kcal})$, in all age groups and in both sexes. However, a consistent observation was the significantly higher dietary fiber intake in females compared to males across the lifecycle. In agreement with our findings, a review by Kiefer et al. [34] concluded that children, adolescents, and adult females tend to consume more dietary fiber compared to their male counterparts. In fact, women and girls have been frequently described as being more health conscious and 
Table 2 Energy and macronutrient intake by gender in the study population $(n=3394)$

\begin{tabular}{|c|c|c|c|c|}
\hline & $\begin{array}{l}\text { Total } \\
(n=3394)\end{array}$ & $\begin{array}{l}\text { Males } \\
(n=1578)\end{array}$ & $\begin{array}{l}\text { Females } \\
(n=1816)\end{array}$ & p-value \\
\hline & \multicolumn{4}{|c|}{ Mean \pm SE } \\
\hline \multicolumn{5}{|c|}{ Children and adolescents (6-19.9 years) } \\
\hline Energy (kcal) & $1999.81 \pm 27.17$ & $2265.55 \pm 38.71$ & $1734.06 \pm 38.48$ & $<0.001$ \\
\hline Carbohydrate (\%EI) & $51.57 \pm 0.34$ & $51.59 \pm 0.47$ & $51.53 \pm 0.47$ & 0.923 \\
\hline Protein (\%EI) & $13.24 \pm 0.13$ & $13.31 \pm 0.17$ & $13.16 \pm 0.17$ & 0.539 \\
\hline Fat (\%El) & $36.11 \pm 0.31$ & $35.78 \pm 0.44$ & $36.44 \pm 0.44$ & 0.300 \\
\hline Saturated fat (\%EI) & $10.86 \pm 0.17$ & $10.76 \pm 0.23$ & $10.95 \pm 0.23$ & 0.565 \\
\hline Oleic Acid (\%EI) & $9.64 \pm 0.2$ & $9.68 \pm 0.27$ & $9.59 \pm 0.27$ & 0.815 \\
\hline Linolenic Acid (\%EI) & $0.13 \pm 0$ & $13 \pm 0$ & $0.13 \pm 0$ & 0.552 \\
\hline Linoleic Acid (\%El) & $4.73 \pm 0.11$ & $4.79 \pm 0.16$ & $4.65 \pm 0.15$ & 0.547 \\
\hline Total Sugar (\%EI) & $12.01 \pm 0.27$ & $11.63 \pm 0.38$ & $12.38 \pm 0.38$ & 0.173 \\
\hline Dietary fibers (g/1000 kcal) & $7.47 \pm 0.13$ & $6.93 \pm 0.19$ & $8.01 \pm 7.65$ & $<0.001$ \\
\hline \multicolumn{5}{|l|}{ Adults ( $20-59.9$ years) } \\
\hline Energy (kcal) & $1983.17 \pm 18.26$ & $2309.95 \pm 29.98$ & $1656.39 \pm 26.07$ & $<0.001$ \\
\hline Carbohydrate (\%EI) & $48.8 \pm 0.25$ & $48.82 \pm 0.42$ & $48.77 \pm 0.36$ & 0.940 \\
\hline Protein (\%El) & $14.66 \pm 0.11$ & $14.68 \pm 0.19$ & $14.63 \pm 0.16$ & 0.868 \\
\hline Fat (\%EI) & $37.09 \pm 0.23$ & $36.45 \pm 0.38$ & $37.73 \pm 0.33$ & 0.017 \\
\hline Saturated fat (\%EI) & $10.84 \pm 0.11$ & $10.45 \pm 0.18$ & $11.24 \pm 0.15$ & 0.002 \\
\hline Oleic Acid (\%EI) & $11.85 \pm 0.15$ & $11.75 \pm 0.25$ & $11.95 \pm 0.22$ & 0.587 \\
\hline Linolenic Acid (\%EI) & $0.15 \pm 0$ & $0.15 \pm 0.01$ & $0.16 \pm 0.01$ & 0.252 \\
\hline Linoleic Acid (\%EI) & $4.96 \pm 0.09$ & $4.87 \pm 0.14$ & $5.05 \pm 0.12$ & 0.368 \\
\hline Total Sugar (\%EI) & $10.85 \pm 0.18$ & $10.69 \pm 0.3$ & $11.01 \pm 0.26$ & 0.455 \\
\hline Dietary fibers (g/1000 kcal) & $8.82 \pm 0.11$ & $8.32 \pm 0.18$ & $9.31 \pm 0.16$ & $<0.001$ \\
\hline \multicolumn{5}{|l|}{ Older adults ( $\geq 60$ years) } \\
\hline Energy (kcal) & $1628.19 \pm 34.1$ & $1861.8 \pm 62.71$ & $1394.59 \pm 63.77$ & $<0.001$ \\
\hline Carbohydrate (\%EI) & $49.48 \pm 0.58$ & $48.28 \pm 1.07$ & $50.68 \pm 1.09$ & 0.187 \\
\hline Protein (\%EI) & $14.88 \pm 0.25$ & $14.54 \pm 0.45$ & $15.22 \pm 0.46$ & 0.380 \\
\hline Fat (\%EI) & $36.27 \pm 0.52$ & $37.35 \pm 0.96$ & $35.2 \pm 0.98$ & 0.189 \\
\hline Saturated fat (\%EI) & $10.5 \pm 0.23$ & $10.42 \pm 0.42$ & $40.58 \pm 0.43$ & 0.826 \\
\hline Oleic Acid (\%EI) & $12.5 \pm 0.34$ & $12.78 \pm 0.63$ & $12.22 \pm 0.64$ & 0.603 \\
\hline Linolenic Acid (\%EI) & $0.17 \pm 0.01$ & $0.15 \pm 0.01$ & $0.18 \pm 0.01$ & 0.167 \\
\hline Linoleic Acid (\%EI) & $4.35 \pm 0.18$ & $4.73 \pm 0.33$ & $3.98 \pm 0.33$ & 0.178 \\
\hline Total Sugar (\%EI) & $10.72 \pm 0.4$ & $11.0 \pm 0.73$ & $10.45 \pm 0.75$ & 0.654 \\
\hline Dietary fibers (g/1000 kcal) & $10.63 \pm 0.26$ & $9.6 \pm 0.47$ & $11.65 \pm 0.48$ & 0.011 \\
\hline
\end{tabular}

Abbreviations: $E l$ energy intake; $g$ grams; $k c a l$ calories; $S E$ standard error

Values in this table represent mean \pm SE

Means were adjusted for variables found to be significantly associated with gender, as per Table 1

* $p$ values were obtained from comparison of adjusted means using ANCOVA

Bolded numbers are significant at $p<0.05$

more inclined to comply with dietary recommendations than their male counterparts [9, 35-38]. However, in contrast to our findings, a recent study conducted in Tunisia did not report any significant differences in dietary fiber intake between males and females [5], while a study conducted in the United Kingdom (UK) showed that, compared to men, women were significantly more likely to have an inadequate fiber intake [4].

In our study, total fat intake ranged between 35.2 and $37.7 \%$ EI, while saturated fat ranged between 10.4 and 
Table 3 Percent contribution of various food groups to total every intake by gender $(n=3394)$

\begin{tabular}{|c|c|c|c|c|}
\hline & \multirow[t]{2}{*}{$\begin{array}{l}\text { Total } \\
(n=3394)\end{array}$} & $\begin{array}{l}\text { Males } \\
(n=1578)\end{array}$ & \multirow[t]{2}{*}{$\begin{array}{l}\text { Females } \\
(n=1816)\end{array}$} & \multirow[t]{2}{*}{$p$-value* } \\
\hline & & Mean $\% \mathrm{El} \pm \mathrm{SE}$ & & \\
\hline \multicolumn{5}{|l|}{ Children and adolescents (6-19.9 years) } \\
\hline Bread & $16.67 \pm 0.44$ & $17.35 \pm 0.62$ & $15.98 \pm 0.62$ & 0.122 \\
\hline Cereals & $14.77 \pm 0.63$ & $15.56 \pm 0.89$ & $13.99 \pm 0.89$ & 0.216 \\
\hline Legumes & $2.69 \pm 0.30$ & $2.48 \pm 0.43$ & $2.9 \pm 0.43$ & 0.484 \\
\hline Starchy Vegetables & $5.14 \pm 0.29$ & $4.85 \pm 0.41$ & $5.44 \pm 0.41$ & 0.311 \\
\hline Vegetables & $4.94 \pm 0.30$ & $4.37 \pm 0.43$ & $5.52 \pm 0.43$ & 0.060 \\
\hline Chips \& Salty Crackers & $4.12 \pm 0.27$ & $4.03 \pm 0.39$ & $4.21 \pm 0.39$ & 0.750 \\
\hline Nuts \& Seeds & $1.67 \pm 0.24$ & $1.22 \pm 0.34$ & $2.11 \pm 0.34$ & 0.066 \\
\hline \multicolumn{5}{|l|}{ Dairy Products } \\
\hline Milk & $1.87 \pm 0.16$ & $1.63 \pm 0.22$ & $2.12 \pm 0.22$ & 0.123 \\
\hline Milk Derivatives & $6.18 \pm 0.29$ & $5.88 \pm 0.41$ & $6.48 \pm 0.41$ & 0.304 \\
\hline Milk Sweetened & $0.31 \pm 0.08$ & $0.32 \pm 0.12$ & $0.31 \pm 0.12$ & 0.956 \\
\hline \multicolumn{5}{|l|}{ Meat, Processed Meat, Poultry, Fish, Eggs } \\
\hline Red Meat & $4.74 \pm 0.33$ & $5.53 \pm 0.47$ & $3.96 \pm 0.47$ & 0.020 \\
\hline Processed Meat & $2.0 \pm 0.20$ & $2.34 \pm 0.29$ & $1.64 \pm 0.29$ & 0.091 \\
\hline Poultry & $3.60 \pm 0.31$ & $3.53 \pm 0.44$ & $3.66 \pm 0.44$ & 0.835 \\
\hline Fish & $0.68 \pm 0.16$ & $0.77 \pm 0.22$ & $0.60 \pm 0.22$ & 0.573 \\
\hline Eggs & $1.07 \pm 0.14$ & $1.14 \pm 0.20$ & $0.98 \pm 0.20$ & 0.578 \\
\hline \multicolumn{5}{|l|}{ Fruits, Total } \\
\hline Whole Fruits & $3.52 \pm 0.20$ & $2.71 \pm 0.28$ & $4.33 \pm 0.28$ & $<0.001$ \\
\hline Fresh Juices (100\% fruit juices) & $0.10 \pm 0.03$ & $0.08 \pm 0.04$ & $0.12 \pm 0.04$ & 0.514 \\
\hline \multicolumn{5}{|l|}{ Sweets \& Added Sugars } \\
\hline Sweets & $9.20 \pm 0.43$ & $8.46 \pm 0.61$ & $9.94 \pm 0.61$ & 0.092 \\
\hline Added Sugars, Jams, Honey, Molasses & $1.82 \pm 0.11$ & $1.77 \pm 0.16$ & $1.88 \pm 0.16$ & 0.617 \\
\hline Sugar Sweetened Beverages & $6.44 \pm 0.24$ & $7.16 \pm 0.34$ & $5.73 \pm 0.34$ & 0.003 \\
\hline Hot Beverages (Coffee, Tea) & $0.11 \pm 0.01$ & $0.11 \pm 0.02$ & $0.1 \pm 0.02$ & 0.888 \\
\hline Alcoholic Beverages & $0.15 \pm 0.06$ & $0.16 \pm 0.08$ & $0.13 \pm 0.08$ & 0.817 \\
\hline Added Fats \& Oils & $4.69 \pm 0.24$ & $4.23 \pm 0.35$ & $5.16 \pm 0.35$ & 0.058 \\
\hline Fast Food & $2.51 \pm 0.29$ & $3.37 \pm 0.42$ & $1.65 \pm 0.42$ & 0.004 \\
\hline Miscellaneous & $1.01 \pm 0.09$ & $0.96 \pm 0.13$ & $1.07 \pm 0.13$ & 0.549 \\
\hline \multicolumn{5}{|l|}{ Adults (20-59.9 years) } \\
\hline Bread & $17.21 \pm 0.34$ & $19.28 \pm 0.54$ & $15.14 \pm 0.49$ & $<0.001$ \\
\hline Cereals & $15.94 \pm 0.50$ & $16.39 \pm 0.79$ & $15.49 \pm 0.72$ & 0.429 \\
\hline Legumes & $3.51 \pm 0.24$ & $4.07 \pm 0.39$ & $2.95 \pm 0.35$ & 0.043 \\
\hline Starchy Vegetables & $3.90 \pm 0.17$ & $3.72 \pm 0.27$ & $4.09 \pm 0.25$ & 0.348 \\
\hline Vegetables & $8.32 \pm 0.28$ & $6.10 \pm 0.45$ & $10.55 \pm 0.41$ & $<0.001$ \\
\hline Chips \& Salty Crackers & $0.93 \pm 0.09$ & $0.74 \pm 0.14$ & $1.12 \pm 0.13$ & 0.059 \\
\hline Nuts \& Seeds & $2.46 \pm 0.20$ & $2.40 \pm 0.31$ & $2.52 \pm 0.28$ & 0.795 \\
\hline \multicolumn{5}{|l|}{ Dairy Products } \\
\hline Milk & $0.99 \pm 0.08$ & $0.70 \pm 0.13$ & $1.29 \pm 0.11$ & 0.001 \\
\hline Milk Derivatives & $7.05 \pm 0.22$ & $6.50 \pm 0.36$ & $7.59 \pm 0.32$ & 0.033 \\
\hline Milk Sweetened & $0.26 \pm 0.06$ & $0.20 \pm 0.09$ & $0.31 \pm 0.08$ & 0.356 \\
\hline
\end{tabular}


Table 3 Percent contribution of various food groups to total every intake by gender $(n=3394)$ (Continued)

\begin{tabular}{|c|c|c|c|c|}
\hline & \multirow[t]{2}{*}{$\begin{array}{l}\text { Total } \\
(n=3394)\end{array}$} & $\begin{array}{l}\text { Males } \\
(n=1578)\end{array}$ & \multirow[t]{2}{*}{$\begin{array}{l}\text { Females } \\
(n=1816)\end{array}$} & \multirow[t]{2}{*}{$p$-value ${ }^{*}$} \\
\hline & & Mean \%El \pm SE & & \\
\hline \multicolumn{5}{|l|}{ Meat, Processed Meat, Poultry, Fish, Eggs } \\
\hline Red Meat & $6.46 \pm 0.29$ & $7.03 \pm 0.46$ & $5.89 \pm 0.42$ & 0.086 \\
\hline Processed Meat & $0.79 \pm 0.08$ & $0.87 \pm 0.12$ & $0.72 \pm 0.11$ & 0.406 \\
\hline Poultry & $4.21 \pm 0.24$ & $4.55 \pm 0.38$ & $3.87 \pm 0.35$ & 0.224 \\
\hline Fish & $1.22 \pm 0.13$ & $1.49 \pm 0.20$ & $0.95 \pm 0.18$ & 0.061 \\
\hline Eggs & $0.74 \pm 0.07$ & $0.86 \pm 0.10$ & $0.61 \pm 0.10$ & 0.095 \\
\hline \multicolumn{5}{|l|}{ Fruits, Total } \\
\hline Whole Fruits & $4.03 \pm 0.15$ & $3.30 \pm 0.23$ & $4.76 \pm 0.21$ & $<0.001$ \\
\hline Fresh Juices (100\% fruit juices) & $0.22 \pm 0.03$ & $0.24 \pm 0.05$ & $0.20 \pm 0.05$ & 0.528 \\
\hline \multicolumn{5}{|l|}{ Sweets \& Added Sugars } \\
\hline Sweets & $6.70 \pm 0.27$ & $4.81 \pm 0.44$ & $8.60 \pm 0.40$ & $<0.001$ \\
\hline Added Sugars, Jams, Honey, Molasses & $1.65 \pm 0.07$ & $1.56 \pm 0.11$ & $1.75 \pm 0.10$ & 0.242 \\
\hline Sugar Sweetened Beverages & $4.0 \pm 0.15$ & $4.42 \pm 0.24$ & $3.57 \pm 0.22$ & 0.012 \\
\hline Hot Beverages (Coffee, Tea) & $0.65 \pm 0.05$ & $0.46 \pm 0.08$ & $0.83 \pm 0.07$ & 0.001 \\
\hline Alcoholic Beverages & $0.74 \pm 0.08$ & $1.30 \pm 0.14$ & $0.19 \pm 0.12$ & $<0.001$ \\
\hline Added Fats \& Oils & $4.32 \pm 0.16$ & $4.62 \pm 0.26$ & $4.01 \pm 0.24$ & 0.110 \\
\hline Fast Food & $2.56 \pm 0.23$ & $3.20 \pm 0.37$ & $1.93 \pm 0.33$ & 0.016 \\
\hline Miscellaneous & $1.15 \pm 0.10$ & $1.20 \pm 0.17$ & $1.10 \pm 0.15$ & 0.675 \\
\hline \multicolumn{5}{|l|}{ Older adults ( $\geq 60$ years) } \\
\hline Bread & $20.82 \pm 0.83$ & $21.88 \pm 1.48$ & $19.77 \pm 1.63$ & 0.424 \\
\hline Cereals & $14.67 \pm 1.07$ & $12.26 \pm 1.90$ & $17.07 \pm 2.09$ & 0.156 \\
\hline Legumes & $3.59 \pm 0.53$ & $4.93 \pm 0.95$ & $2.25 \pm 1.04$ & 0.112 \\
\hline Starchy Vegetables & $2.68 \pm 0.34$ & $2.89 \pm 0.61$ & $2.46 \pm 0.66$ & 0.686 \\
\hline Vegetables & $10.55 \pm 0.68$ & $8.02 \pm 1.22$ & $13.08 \pm 1.33$ & 0.020 \\
\hline Chips \& Salty Crackers & $0.22 \pm 0.12$ & $0.34 \pm 0.22$ & $0.10 \pm 0.24$ & 0.533 \\
\hline Nuts \& Seeds & $0.99 \pm 0.27$ & $2.07 \pm 0.49$ & 0 & N/A \\
\hline \multicolumn{5}{|l|}{ Dairy Products } \\
\hline Milk & $1.50 \pm 0.22$ & $1.06 \pm 0.39$ & $1.94 \pm 0.43$ & 0.203 \\
\hline Milk Derivatives & $9.02 \pm 0.52$ & $7.70 \pm 0.93$ & $10.34 \pm 1.02$ & 0.110 \\
\hline Milk Sweetened & $0.30 \pm 0.15$ & $0.32 \pm 0.27$ & $0.28 \pm 0.29$ & 0.935 \\
\hline \multicolumn{5}{|l|}{ Meat, Processed Meat, Poultry, Fish, Eggs } \\
\hline Red Meat & $7.54 \pm 0.65$ & $8.75 \pm 1.16$ & $6.33 \pm 1.27$ & 0.242 \\
\hline Processed Meat & $0.51 \pm 0.19$ & $0.55 \pm 0.33$ & $0.46 \pm 0.37$ & 0.890 \\
\hline Poultry & $3.19 \pm 0.42$ & $3.35 \pm 0.75$ & $3.03 \pm 0.82$ & 0.813 \\
\hline Fish & $0.45 \pm 0.13$ & $0.49 \pm 0.22$ & $0.40 \pm 0.25$ & 0.823 \\
\hline Eggs & $0.82 \pm 0.20$ & $0.75 \pm 0.36$ & $0.89 \pm 0.39$ & 0.825 \\
\hline \multicolumn{5}{|l|}{ Fruits, Total } \\
\hline Whole Fruits & $7.38 \pm 0.42$ & $6.49 \pm 0.74$ & $8.26 \pm 0.81$ & 0.180 \\
\hline Fresh Juices (100\% fruit juices) & $0.26 \pm 0.08$ & $0.23 \pm 0.14$ & $0.30 \pm 0.15$ & 0.800 \\
\hline \multicolumn{5}{|l|}{ Sweets \& Added Sugars } \\
\hline Sweets & $4.09 \pm 0.53$ & $5.05 \pm 0.95$ & $3.12 \pm 1.04$ & 0.250 \\
\hline Added Sugars, Jams, Honey, Molasses & $1.49 \pm 0.16$ & $1.43 \pm 0.28$ & $1.54 \pm 0.31$ & 0.828 \\
\hline
\end{tabular}


Table 3 Percent contribution of various food groups to total every intake by gender $(n=3394)$ (Continued)

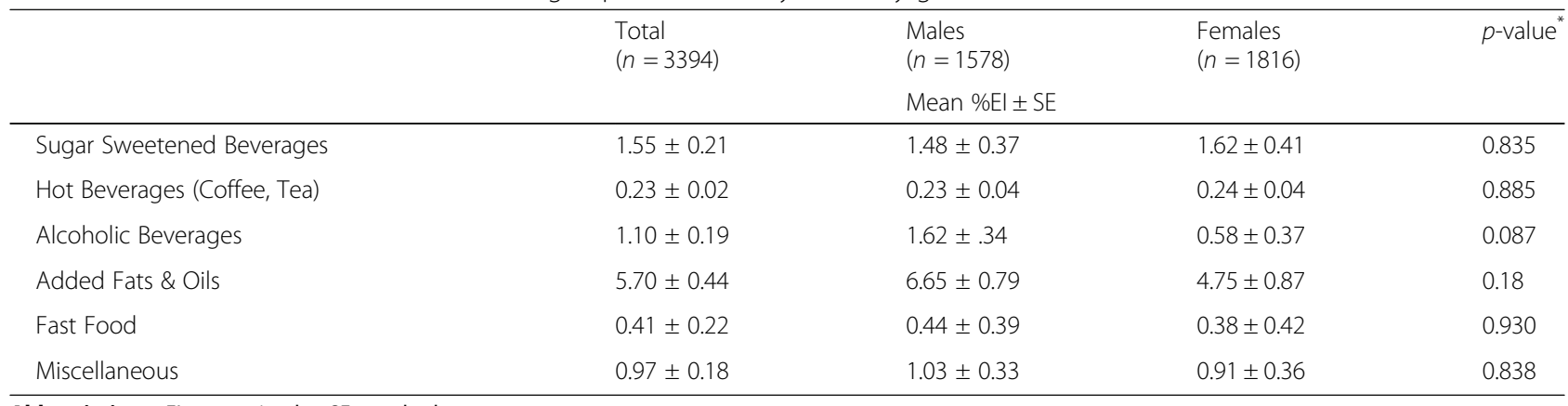

Abbreviations: $E$ l energy intake; SE standard error

Values in this table represent mean \pm SE

Means were adjusted for variables found to be significantly associated with gender, as per Table 1

" $p$ values were obtained from comparison of adjusted means using ANCOVA

Bolded numbers are significant at $p<0.05$

$11.2 \%$ EI, thus exceeding, in all age groups and in both sexes, the World Health Organization (WHO) upper limit of $35 \%$ EI for total fat and 8-10\% EI for saturated fat [39]. However, significant sex-based differences in the macronutrients' contributions to energy intake were observed. More specifically, adolescent girls and adult women (20-60 years) were found to have significantly higher intakes of total fat compared to males, but these differences were not observed in younger children nor in older adults. Noteworthy is the higher intake of saturated fat that was also observed among adult women compared to men. Similar to our findings, the $2012 \mathrm{Na}$ tional Diet and Nutrition Survey in the UK reported that women consume more fat and saturated fat than their male counterparts [40]. A prospective study conducted on a large sample of adults in the UK has also shown that, compared to men, women were more likely to have intakes that exceeded recommendations for total fat and saturated fat [4]. Our findings contrast with data stemming from 22 European countries [9], as well as from Tunisia [5], which did not report any significant sexbased differences in total fat intake. Our findings are also in contrast with studies reporting women as often choosing lower fat foods in an effort to make heathier food choices [9, 34].

In line with the observed differences in macronutrients' intakes, sex-based disparities in food consumption patterns were observed. Our results underline a higher intake of fruits, vegetables, and milk in females compared to men, while showing that men had a higher consumption of soft drinks, red meat, fast food, bread, and alcohol. These findings are in agreement with previous studies conducted in Lebanon showing that males were more likely to adhere to the Western dietary pattern, while females had higher adherence to the Traditional Lebanese dietary pattern, which has been described as a variant of the Mediterranean diet with fruits, vegetables, and dairy consumption being among its characteristic hallmarks [22, 23, 41, 42]. Our findings are also in agreement with those reported from studies conducted in other parts of the world [6, 9, 43]. For instance, in Tunisia, another country undergoing the nutrition transition, women were found to consume more fruit and less soft drinks and red meat compared to men. Earlier studies have described significant gender differences in opinions and behaviors related to dietary and food choices [9]. These studies reported that men choose fewer high-fiber foods, eat less fruits and vegetables, while consuming more soft drinks and high starch foods such as bread, while women tend to consume a higher number of portions of fruits and vegetables compared to men $[6,9,43-50]$. Fruits and vegetables are rich sources of dietary fiber, antioxidants, and phytochemicals and hence dietary guidelines and recommendations have consistently encouraged a higher consumption of these food groups [51, 52]. The recent Global Burden of Disease Study (GBD) [53] showed that the optimal intake of fruit in relation to all-cause mortality falls within the range of $200-300 \mathrm{~g} /$ day and the optimal intake of vegetables between 290 and $430 \mathrm{~g} /$ day. Thus in our study, despite females having higher intakes of fruits (and of vegetables in the elderly age group) than males, their intake levels ranging between 102 and $136 \mathrm{~g} /$ day for fruits and between 166 and $188 \mathrm{~g}$ /day for vegetables (data not shown) remain low in comparison with optimal intake levels [53]. In addition, despite the significantly lower intake of red meat in females compared to males (except among the elderly age group), the consumption of red meat among Lebanese girls and women was not low compared to dietary recommendations, unlike what is usually reported from developing countries [8]. In fact, red meat intakes in females, which ranged from 27 to 
Table 4 Micronutrient intake (expressed as means \pm SE and percentage consuming $<2 / 3 r d$ RDA) by gender $(n=3394)$

\begin{tabular}{|c|c|c|c|c|}
\hline & $\begin{array}{l}\text { Total } \\
(n=3394)\end{array}$ & $\begin{array}{l}\text { Males } \\
(n=1578)\end{array}$ & $\begin{array}{l}\text { Females } \\
(n=1816)\end{array}$ & p-value \\
\hline \multicolumn{5}{|c|}{ Children and adolescents (6-19.9 years) } \\
\hline \multicolumn{5}{|l|}{ Calcium (mg) } \\
\hline Mean \pm SE & $593.67 \pm 12.4$ & $657.35 \pm 17.65$ & $529.98 \pm 17.57$ & $<0.001$ \\
\hline Below 2/3rd RDA, n (\%) & $669(77.3)$ & $311(71.2)$ & $358(83.6)$ & $<0.001$ \\
\hline \multicolumn{5}{|l|}{ Iron (mg) } \\
\hline Mean \pm SE & $9.93 \pm 0.21$ & $11.05 \pm 0.3$ & $8.81 \pm 0.3$ & $<0.001$ \\
\hline Below 2/3rd RDA, n (\%) & $315(36.4)$ & $108(24.7)$ & $207(48.4)$ & $<0.001$ \\
\hline \multicolumn{5}{|l|}{ Zinc (mg) } \\
\hline Mean \pm SE & $7.84 \pm 0.15$ & $8.88 \pm 0.22$ & $6.8 \pm 0.21$ & $<0.001$ \\
\hline Below 2/3rd RDA, n (\%) & $230(26.6)$ & $87(20)$ & $143(33.4)$ & $<0.001$ \\
\hline \multicolumn{5}{|l|}{ Vitamin $\mathrm{A}(\mathrm{RAE}, \mu \mathrm{g})$} \\
\hline Mean \pm SE & $788.4 \pm 68.91$ & $931.87 \pm 98.1$ & $644.93 \pm 97.63$ & 0.039 \\
\hline Below 2/3rd RDA, n (\%) & $478(55.3)$ & $238(54.6)$ & $240(56.1)$ & 0.660 \\
\hline \multicolumn{5}{|l|}{ Vitamin C (mg) } \\
\hline Mean \pm SE & $82.86 \pm 2.43$ & $87.14 \pm 3.46$ & $78.59 \pm 3.45$ & 0.082 \\
\hline Below 2/3rd RDA, n (\%) & $226(26.2)$ & $108(24.8)$ & $118(27.7)$ & 0.329 \\
\hline \multicolumn{5}{|l|}{ Vitamin B12 $(\mu \mathrm{g})$} \\
\hline Mean \pm SE & $3.63 \pm 0.35$ & $4.58 \pm 0.5$ & $2.68 \pm 0.5$ & 0.008 \\
\hline Below 2/3rd RDA, n (\%) & $309(35.7)$ & $130(29.7)$ & $179(41.8)$ & $<0.001$ \\
\hline \multicolumn{5}{|l|}{ Adults (20-59.9 years) } \\
\hline \multicolumn{5}{|l|}{ Calcium (mg) } \\
\hline Mean \pm SE & $603.48 \pm 7.93$ & $669.8 \pm 13.02$ & $537.16 \pm 11.32$ & $<0.001$ \\
\hline Below 2/3rd RDA, n (\%) & $1392(66.1)$ & $525(56.7)$ & $867(73.5)$ & $<0.001$ \\
\hline \multicolumn{5}{|l|}{ Iron (mg) } \\
\hline Mean \pm SE & $10.68 \pm 0.14$ & $12.13 \pm 0.23$ & $9.23 \pm 0.2$ & $<0.001$ \\
\hline Below 2/3rd RDA, n (\%) & $948(45.0)$ & $125(13.5)$ & $823(69.8)$ & $<0.001$ \\
\hline \multicolumn{5}{|l|}{ Zinc (mg) } \\
\hline Mean \pm SE & $8.8 \pm 0.12$ & $10.18 \pm 0.2$ & $7.41 \pm 0.18$ & $<0.001$ \\
\hline Below 2/3rd RDA, n (\%) & $698(33.2)$ & $245(26.5)$ & $453(38.4)$ & $<0.001$ \\
\hline \multicolumn{5}{|l|}{ Vitamin A (RAE, $\mu \mathrm{g})$} \\
\hline Mean \pm SE & $842.36 \pm 47.0$ & $876.34 \pm 77.18$ & $808.37 \pm 67.1$ & 0.536 \\
\hline Below 2/3rd RDA, n (\%) & $1339(63.6)$ & $608(65.7)$ & $731(62.0)$ & 0.084 \\
\hline \multicolumn{5}{|l|}{ Vitamin C (mg) } \\
\hline Mean \pm SE & $83.44 \pm 1.56$ & $91.84 \pm 2.56$ & $75.04 \pm 2.22$ & $<0.001$ \\
\hline Below 2/3rd RDA, n (\%) & $850(41.4)$ & $383(41.4)$ & $467(41.4)$ & 0.972 \\
\hline \multicolumn{5}{|l|}{ Vitamin B12 $(\mu \mathrm{g})$} \\
\hline Mean \pm SE & $5.0 \pm 0.37$ & $5.85 \pm 0.6$ & $4.06 \pm 0.53$ & 0.038 \\
\hline Below 2/3rd RDA, n (\%) & $908(43.1)$ & $327(35.3)$ & $581(49.3)$ & $<0.001$ \\
\hline \multicolumn{5}{|l|}{ Older adults ( $\geq 60$ years) } \\
\hline \multicolumn{5}{|l|}{ Calcium (mg) } \\
\hline Mean \pm SE & $541.31 \pm 14.52$ & $567.81 \pm 26.69$ & $514.82 \pm 27.15$ & 0.243 \\
\hline Below 2/3rd RDA, n (\%) & $336(79.2)$ & $162(75.3)$ & $174(83.3)$ & 0.045 \\
\hline
\end{tabular}

Iron (mg) 
Table 4 Micronutrient intake (expressed as means \pm SE and percentage consuming $<2 / 3 r d$ RDA) by gender $(n=3394)$ (Continued)

\begin{tabular}{|c|c|c|c|c|}
\hline & $\begin{array}{l}\text { Total } \\
(n=3394)\end{array}$ & $\begin{array}{l}\text { Males } \\
(n=1578)\end{array}$ & $\begin{array}{l}\text { Females } \\
(n=1816)\end{array}$ & $p$-value \\
\hline Mean \pm SE & $9.22 \pm 0.33$ & $10.08 \pm 0.6$ & $8.36 \pm 0.61$ & 0.090 \\
\hline Below 2/3rd RDA, n (\%) & $123(29.0)$ & $46(21.4)$ & 77 (36.8) & $<0.001$ \\
\hline \multicolumn{5}{|l|}{ Zinc (mg) } \\
\hline Mean \pm SE & $7.41 \pm 0.2$ & $8.39 \pm 0.37$ & $6.42 \pm 0.38$ & 0.002 \\
\hline Below 2/3rd RDA, n (\%) & $178(42.0)$ & 85 (39.5) & $93(44.5)$ & 0.301 \\
\hline \multicolumn{5}{|l|}{ Vitamin A (RAE, $\mu \mathrm{g})$} \\
\hline Mean \pm SE & $641.17 \pm 63.27$ & $678.58 \pm 116.36$ & $603.77 \pm 116.36$ & 0.705 \\
\hline Below 2/3rd RDA, n (\%) & $286(67.5)$ & $156(72.6)$ & $130(62.2)$ & 0.023 \\
\hline \multicolumn{5}{|l|}{ Vitamin C (mg) } \\
\hline Mean \pm SE & $77.75 \pm 4.14$ & $75.85 \pm 7.61$ & $79.65 \pm 7.73$ & 0.769 \\
\hline Below 2/3rd RDA, n (\%) & $186(45.1)$ & $112(52.1)$ & $74(37.6)$ & 0.003 \\
\hline \multicolumn{5}{|l|}{ Vitamin B12 ( $\mu \mathrm{g})$} \\
\hline Mean \pm SE & $3.57 \pm 0.54$ & $4.35 \pm 1.0$ & $2.79 \pm 1.01$ & 0.356 \\
\hline Below 2/3rd RDA, n (\%) & $212(50.0)$ & $102(47.4)$ & $110(52.6)$ & 0.286 \\
\hline
\end{tabular}

Abbreviations: $\mu g$ micrograms; $m g$ milligrams; RAE Retinol Activity Equivalents; RDA Recommended Daily Allowance; SE standard error Values in this table represent mean \pm SE for continuous variables and $n(\%)$ for categorical variables

Means were adjusted for variables found to be significantly associated with gender, as per Table 1

" $p$-values were obtained from comparison of adjusted means using ANCOVA for continuous variables and from chi square for categorical variables Bolded numbers are significant at $p<0.05$

$32 \mathrm{~g} /$ day (vs 52-60 g/day for males) was within the upper limit specified by the GBD study (18-27 g/day) (data not shown) [53]. The fact that, in our study, women had a higher intake of sweets than men is also in line with findings reported by previous studies [5, 54], which have argued that men tend to prefer "hot hearty" food such as steak, while women tend to prefer sweet snacks, such as chocolate and ice cream [54]. Sweets have in fact been suggested to be culturally associated with femininity [55], while meat consumption is far more commonly associated with masculinity [56]. The observed higher sweets intake may explain the fact that a significantly higher proportion of females exceeded the upper limit of total sugar intake (25\% EI) compared to men (data not shown), although the average total sugar intake did not differ between both sexes. Sweets can also be an important source of "hidden fat", which may also explain the observed higher intakes of fat and saturated fat in women compared to men.

In agreement with evidence stemming from developing countries [8], the present study showed that women and girls in Lebanon have lower micronutrient intakes compared to their male counterparts and that higher proportions of females may be at risk of micronutrient inadequacies, including calcium, iron, zinc, and vitamin B12. In contrast to our findings, data stemming from Western societies have documented strong similarities in micronutrient intakes between both sexes [9]. The micronutrient inadequacies characterized in our study are in line with the priority micronutrients identified by the WHO for children and women of childbearing age in the EMR [57] and with those documented in previous studies in Lebanon. For instance, a cross-sectional survey by $\mathrm{Al}$ Khatib et al. of 470 Lebanese women (aged 15-45 years), reported that $16 \%$ were anemic $(\mathrm{Hb}<12 \mathrm{~g} / \mathrm{dL})$, $27.2 \%$ were iron deficient (ferritin $<15 \mu \mathrm{g} / \mathrm{L}$ ), and $7.7 \%$ were diagnosed with iron deficiency anemia [58]. In our study, the observed high proportions of women and girls not meeting 2/3rd of the RDA for several micronutrients is of concern given the impact that micronutrient inadequacies may have on physiological performance, risk for chronic disease [59-61], as well as the cycle of micronutrient inadequacies across the life span [59]. Importantly, women and girls with these nutritional inadequacies and deficits are likely to have lower educational attainment and work capability, thus contributing to further inequalities in social roles between genders [59].

Existent gender inequities and differences in social roles may also contribute to disparities in food and dietary intakes and may, at least partly, explain the observed sex-based dietary differences in our study. Despite its modernized flavor, Lebanon remains a patriarchal culture with a strong set of traditional social values, where boys tend to be given entitlement over their sisters from early childhood [62]. In a study conducted in Lebanon about perceptions of the ideal woman, the most 
frequently mentioned attributes, were 'being a good housewife and mother', 'sacrificing', 'devoted to her family', whereas attributes related to a woman's personality or education were rarely cited [63]. These culturally dominant attributes and social roles may in fact influence the eating behavior of women and girls in Lebanon. For instance, women who do not work outside the home or who are mostly in charge of preparing meals in the household may receive more food stimuli than men, including stimuli for unhealthy foods such as sweets [5, 64]. Women may also "sacrifice" by eating less from certain foods groups, in favor of their children. A recent study by Jomaa et al. (2017) showed that, in an effort to ensure that their children are well-fed, Lebanese mothers may consume less animal-based foods such as meats and may thus have an inadequate intake of several micronutrients including iron, zinc and vitamin B12 [65, 66]. Other factors such as lower income, insufficient professional insertion, and limited decision-making within the household may also contribute to differences in dietary choices between women and men, and between boys and girls in Lebanon [67].

Overall, this study identified and characterized sexbased differences in dietary intakes and food consumption patterns in a nutrition transition context. Despite the favorable observation of higher intakes of dietary fiber, fruits, and vegetables in females compared to males, dietary intakes in women and girls remained inadequate in several micronutrients of public health concern while also being high in total and saturated fat. These observed sex-based differences place Lebanon somewhere between developed and developing countries in terms of dietary disparities between sexes, sharing the micronutrients inequalities with developing countries, while also sharing the higher fruit, vegetables, and fiber intakes with Western societies. Of interest in our study is the fact that sex-based differences in macronutrient intakes and food consumption patterns were the most noticeable in the adolescent and adult years, a timeframe that includes women's reproductive years. These findings highlight the need for culture-specific interventions aimed at improving nutrition for women and adolescent girls as this would lay the foundation not only for their future education, productivity, and economic empowerment, but also for the health of future generations [1]. The study findings have also highlighted higher intakes of Westernized "modern" foods in males compared to females, including higher intakes of soft drinks, red and processed meat, and alcohol. Adherence to a Westernized diet has been associated with increased risk for obesity and several non-communicable diseases, thus highlighting the need for context-specific interventions aimed at promoting healthier dietary patterns in Lebanese boys and men [22, 23, 41, 42].
The present study has several strengths. The study is based on an individual food consumption survey that was conducted on a nationally representative sample of children, adolescents, and adults. The survey has covered a wide age range, permitting to examine sex-based disparities in dietary intakes across the lifespan. To reduce judgmental communication and minimize social desirability bias, the 24-HRs assessment was administered by trained nutritionists who received extensive training prior to data collection. The results of the study ought however to be considered in light of the following limitations. First, the investigation of food consumption and nutrient intakes was based on the collection of one 24$\mathrm{HR}$, which may not be representative of dietary intakes at the individual level. However, despite its well-known limitations, such as reliance on memory and day-to-day variation, the 24-HR may provide accurate estimates of energy intake and nutrient intakes at the population level [68]. In the present study, dietary information was collected by the multiple pass 24-HR approach, which was shown to improve the accuracy of dietary intake estimates in children, adolescents, and adults [69-71]. Second, it is important to mention that, in Lebanon, there exists no food composition database. Therefore, the USDA database of the Nutritionist Pro was used for dietary analysis including that of traditional dishes and mixed recipes. Although this limitation could have affected the absolute estimates of nutrient intakes, it would have had limited effects on sex-specific differences. Lastly, some of the observed differences, though statistically significant, were rather small in magnitude and hence their clinical implications may be limited.

\section{Conclusion}

Based on an individual national nutrition survey conducted in Lebanon, this study identified sex-specific priorities that ought to be tackled by nutrition interventions aimed at promoting healthier dietary patterns and improving nutrition in both sexes. The fact that, in our study, disparities in food consumption patterns and nutrient intakes were mostly noticeable in the adolescent and adult years, and hence women's reproductive years, call for concerted efforts to improve the diets of women and girls, putting more emphasis on the quality of food consumed and not only its quantity. Acknowledging that gender inequality crosscuts with inadequate health care, insufficient education, and limited income, sex-based differences in diet and food consumption ought to be placed at the heart of the United $\mathrm{Na}$ tions' SDGs on gender equality and women's empowerment, nudging policy makers to recognize that ending hunger cannot be achieved unless all women can consume enough food with adequate nutrients [72]. 


\section{Appendix}

Table 5 Food items included in each of the food groups considered in this study

\begin{tabular}{ll}
\hline Food Groups & Composition \\
\hline Bread & All kinds of bread \\
Cereals & $\begin{array}{l}\text { Cereals, rice, rice-based dishes, pasta, bulgur, } \\
\text { pizza }\end{array}$ \\
Legumes & All kinds of legumes, legume-based dishes \\
Starchy Vegetables & $\begin{array}{l}\text { Potatoes (including potato-based dishes), } \\
\text { pumpkin, sweet corn }\end{array}$ \\
Vegetables & $\begin{array}{l}\text { Raw vegetables (including all kinds of salads } \\
\text { \& all kinds of vegetables), } \\
\text { cooked vegetables, vegetable-based trad- } \\
\text { itional dishes, } \\
\text { canned vegetables (including heart of palm, } \\
\text { carrots, tomato juice, artichoke) }\end{array}$
\end{tabular}

\section{Chips \& Salty Crackers Chips, pretzels, popcorn}

Nuts \& Seeds

\section{Dairy Products}

$\begin{array}{ll}\text { Milk } & \text { All kinds of milk (including infant formula) } \\ \text { Milk derivatives } & \begin{array}{l}\text { All kinds of cheese, yoghurt, laban,yoghurt- } \\ \text { based dishes }\end{array} \\ \text { Milk sweetened } & \begin{array}{l}\text { Milk-based puddings, frozen yoghurt, fruit } \\ \text { yoghurt }\end{array}\end{array}$

Meat, Processed Meat, Poultry, Fish, Eggs

$\begin{array}{ll}\text { Red meat } & \text { Meat and meat organs } \\ \text { Processed meat } & \text { Processed meat } \\ \text { Poultry } & \text { Poultry and poultry organs } \\ \text { Fish } & \text { All kinds of seafood } \\ \text { Eggs } & \text { Eggs }\end{array}$

\section{Fruits, Total}

Whole fruits

$$
\text { Fruits and dried fruits }
$$

Fresh juices (100\%

fruit juices)

All kinds of fresh fruits

\section{Sweets \& Added Sugars}

Sweets

Pastries, candies, biscuits, cakes, traditional sweets, ice-cream

Added sugars, jams, Added sugars, jams, honey, molasses

honey, molasses

\section{Sugar Sweetened Sweetened juices, regular soft drinks \\ Beverages}

Hot Beverages (Coffee, All kinds of coffee and tea

Tea)

Alcoholic Beverages

Added Fats \& Oils

Fast Food

Miscellaneous
Gin, rum, whiskey, arak, vodka, wine, beer Olive oil, olives, avocado, sesame butter, all kinds of oils, mayonnaise, salad dressings, animal-based fat

All kinds of fast food

Pickles, soups, broth
Table 6 Energy and macronutrient intake by gender among adolescents (12-19.9 years) $(n=486)^{*}$

\begin{tabular}{|c|c|c|c|c|}
\hline & $\begin{array}{l}\text { Total }{ }^{\mathbb{P}} \\
(\boldsymbol{n}=486)\end{array}$ & $\begin{array}{l}\text { Males } \mathbb{P} \\
(\boldsymbol{n}=237)\end{array}$ & $\begin{array}{l}\text { Females }^{\mathbb{P}} \\
(\boldsymbol{n}=249)\end{array}$ & $\begin{array}{l}p- \\
\text { value }\end{array}$ \\
\hline \multicolumn{5}{|c|}{ Mean \pm SE } \\
\hline Energy (kcal) & $2185.63 \pm 39.28$ & $2555.37 \pm 56.86$ & $1815.88 \pm 55.45$ & $\begin{array}{l}< \\
0.001\end{array}$ \\
\hline $\begin{array}{l}\text { Carbohydrate } \\
\text { (\%EI) }\end{array}$ & $51.43 \pm 0.46$ & $51.86 \pm 0.67$ & $51.01 \pm 0.65$ & 0.366 \\
\hline Protein (\%EI) & $13.37 \pm 0.17$ & $13.58 \pm 0.25$ & $13.16 \pm 0.25$ & 0.234 \\
\hline Fat (\%EI) & $36.03 \pm 0.42$ & $35.03 \pm 0.61$ & $37.02 \pm 0.6$ & 0.020 \\
\hline $\begin{array}{l}\text { Saturated fat } \\
(\% E I)\end{array}$ & $10.69 \pm 0.2$ & $10.38 \pm 0.28$ & $10.1 \pm 0.28$ & 0.125 \\
\hline $\begin{array}{l}\text { Oleic Acid } \\
\text { (\%EI) }\end{array}$ & $9.93 \pm 0.27$ & $10.05 \pm 0.39$ & $9.82 \pm 0.38$ & 0.684 \\
\hline $\begin{array}{l}\text { Linolenic Acid } \\
\text { (\%EI) }\end{array}$ & $0.13 \pm 0$ & $0.13 \pm 0.01$ & $0.13 \pm 0.01$ & 0.582 \\
\hline $\begin{array}{l}\text { Linoleic Acid } \\
(\% \mathrm{EI})\end{array}$ & $4.78 \pm 0.16$ & $4.74 \pm 0.22$ & $4.81 \pm 0.22$ & 0.820 \\
\hline $\begin{array}{l}\text { Total Sugar } \\
\text { (\%EI) }\end{array}$ & $12.13 \pm 0.37$ & $11.77 \pm 0.54$ & $12.5 \pm 0.52$ & 0.340 \\
\hline $\begin{array}{l}\text { Dietary fibers } \\
\text { (g/1000 kcal) }\end{array}$ & $7.55 \pm 0.19$ & $6.89 \pm 0.27$ & $8.21 \pm 0.27$ & 0.001 \\
\hline
\end{tabular}

Abbreviations: $E$ l energy intake; $g$ grams; kcal calories; SE standard error * Only including participants with completed dietary intake, and excluding those with Kcal outliers

" Analysis of covariance (ANCOVA) was performed after adjusting for age and governorates

Bolded numbers are significant at $p<0.05$ 
Table 7 Daily food consumption (\% El) contributed by food groups according to gender, among adolescents (12-19.9 years) $(n=476)^{*}$

\begin{tabular}{|c|c|c|c|c|}
\hline & \multirow[t]{2}{*}{$\begin{array}{l}\text { Total } \mathbb{P} \\
(n=476)\end{array}$} & $\begin{array}{l}\text { Males } \mathbb{P} \\
(n=235)\end{array}$ & $\begin{array}{l}\text { Females } \mathbb{P} \\
(n=241)\end{array}$ & \multirow[t]{2}{*}{$p$-value } \\
\hline & & \multicolumn{2}{|l|}{ Mean \%El \pm SE } & \\
\hline Bread & $16.0 \pm 0.58$ & $17.31 \pm 0.84$ & $14.68 \pm 0.83$ & 0.028 \\
\hline Cereals & $15.87 \pm 0.88$ & $17.52 \pm 1.27$ & $14.23 \pm 1.25$ & 0.069 \\
\hline Legumes & $2.71 \pm 0.39$ & $2.43 \pm 0.57$ & $2.98 \pm 0.56$ & 0.491 \\
\hline Starchy Vegetables & $4.70 \pm 0.34$ & $4.20 \pm 0.48$ & $5.21 \pm 0.48$ & 0.145 \\
\hline Vegetables & $5.64 \pm 0.44$ & $4.99 \pm 0.63$ & $6.30 \pm 0.63$ & 0.148 \\
\hline Chips \& Salty Crackers & $3.79 \pm 0.35$ & $3.12 \pm 0.50$ & $4.46 \pm 0.49$ & 0.059 \\
\hline Nuts \& Seeds & $1.80 \pm 0.34$ & $0.91 \pm 0.48$ & $2.67 \pm 0.48$ & 0.010 \\
\hline \multicolumn{5}{|l|}{ Dairy Products } \\
\hline Milk & $1.06 \pm 0.14$ & $0.82 \pm 0.21$ & $1.29 \pm 0.20$ & 0.102 \\
\hline Milk Derivatives & $6.22 \pm 0.38$ & $6.13 \pm 0.54$ & $6.30 \pm 0.54$ & 0.825 \\
\hline Milk Sweetened & $0.11 \pm 0.05$ & $0.12 \pm 0.07$ & $0.10 \pm 0.07$ & 0.821 \\
\hline \multicolumn{5}{|l|}{ Meat, Processed Meat, Poultry, Fish, Eggs } \\
\hline Red Meat & $4.92 \pm 0.46$ & $5.81 \pm 0.66$ & $4.04 \pm 0.66$ & 0.061 \\
\hline Processed Meat & $1.45 \pm 0.24$ & $2.04 \pm 0.34$ & $0.86 \pm 0.34$ & 0.015 \\
\hline Poultry & $4.27 \pm 0.46$ & $3.91 \pm 0.66$ & $4.63 \pm 0.65$ & 0.441 \\
\hline Fish & $0.86 \pm 0.25$ & $1.06 \pm 0.36$ & $0.66 \pm 0.35$ & 0.424 \\
\hline Eggs & $0.95 \pm 0.20$ & $1.13 \pm 0.28$ & $0.77 \pm 0.28$ & 0.370 \\
\hline \multicolumn{5}{|l|}{ Fruits, Total } \\
\hline Whole Fruits & $3.16 \pm 0.26$ & $2.36 \pm 0.38$ & $3.97 \pm 0.37$ & 0.003 \\
\hline Fresh Juices (100\% fruit juices) & $0.13 \pm 0.05$ & $0.08 \pm 0.07$ & $0.17 \pm 0.07$ & 0.371 \\
\hline \multicolumn{5}{|l|}{ Sweets \& Added Sugars } \\
\hline Sweets & $8.98 \pm 0.58$ & $7.69 \pm 0.83$ & $10.26 \pm 0.82$ & 0.030 \\
\hline Added Sugars, Jams, Honey, Molasses & $1.54 \pm 0.14$ & $1.47 \pm 0.20$ & $1.61 \pm 0.20$ & 0.617 \\
\hline Sugar Sweetened Beverages & $6.58 \pm 0.32$ & $6.99 \pm 0.46$ & $6.17 \pm 0.45$ & 0.202 \\
\hline Hot Beverages (Coffee, Tea) & $0.13 \pm 0.02$ & $0.15 \pm 0.03$ & $0.12 \pm 0.03$ & 0.562 \\
\hline Alcoholic Beverages & $0.24 \pm 0.09$ & $0.27 \pm 0.14$ & $0.22 \pm 0.13$ & 0.814 \\
\hline Added Fats \& Oils & $4.63 \pm 0.32$ & $4.14 \pm 0.46$ & $5.12 \pm 0.45$ & 0.133 \\
\hline Fast Food & $3.35 \pm 0.46$ & $4.54 \pm 0.66$ & $2.15 \pm 0.65$ & 0.011 \\
\hline Miscellaneous & $0.93 \pm 0.11$ & $0.81 \pm 0.16$ & $1.05 \pm 0.16$ & 0.298 \\
\hline
\end{tabular}

Abbreviations: $E$ l energy intake; SE standard error

* Only including participants with completed dietary intake, and excluding those with Kcal outliers

"Analysis of covariance (ANCOVA) was performed after adjusting for age and governorates

Bolded numbers are significant at $p<0.05$ 
Table 8 Micronutrient intake among adolescents (12-19.9 years) $(n=495)^{*}$

\begin{tabular}{|c|c|c|c|c|}
\hline & $\begin{array}{l}\text { Total }{ }^{\mathbb{P}} \\
(\mathrm{n}=495)\end{array}$ & $\begin{array}{l}\text { Males } \mathbb{P} \\
(\boldsymbol{n}=244)\end{array}$ & $\begin{array}{l}\text { Females } \mathbb{P} \\
(\boldsymbol{n}=251)\end{array}$ & $p$-value \\
\hline \multicolumn{5}{|l|}{ Calcium (mg) } \\
\hline Mean \pm SE & $634.28 \pm 16.98$ & $745.03 \pm 24.55$ & $523.52 \pm 24.0$ & $<0.001$ \\
\hline Below 2/3rd RDA, n (\%) & $377(76.2)$ & $160(65.6)$ & $217(86.5)$ & $<0.001$ \\
\hline \multicolumn{5}{|l|}{ Iron (mg) } \\
\hline Mean \pm SE & $10.96 \pm 0.31$ & $12.64 \pm 0.44$ & $9.28 \pm 0.43$ & $<0.001$ \\
\hline Below 2/3rd RDA, n (\%) & $185(37.4)$ & $41(16.8)$ & $144(57.4)$ & $<0.001$ \\
\hline \multicolumn{5}{|l|}{ Zinc (mg) } \\
\hline Mean \pm SE & $8.65 \pm 0.22$ & $10.13 \pm 0.32$ & $7.18 \pm 0.31$ & $<0.001$ \\
\hline Below 2/3rd RDA, n (\%) & $141(28.5)$ & $45(18.5)$ & $96(38.2)$ & $<0.001$ \\
\hline \multicolumn{5}{|l|}{ Vitamin $A(R A E, \mu g)$} \\
\hline Mean \pm SE & $882.63 \pm 102.14$ & $1118.0 \pm 147.67$ & $647.35 \pm 144.31$ & 0.025 \\
\hline Below 2/3rd RDA, n (\%) & $315(63.8)$ & $156(64.2)$ & $159(63.3)$ & 0.844 \\
\hline \multicolumn{5}{|l|}{ Vitamin C (mg) } \\
\hline Mean \pm SE & $81.68 \pm 3.1$ & $85.83 \pm 4.49$ & $77.52 \pm 4.38$ & 0.190 \\
\hline Below 2/3rd RDA, n (\%) & $161(32.7)$ & 77 (31.7) & $84(33.7)$ & 0.629 \\
\hline \multicolumn{5}{|l|}{ Vitamin B12 $(\mu \mathrm{g})$} \\
\hline Mean \pm SE & $4.35 \pm 0.56$ & $6.14 \pm 0.82$ & $2.57 \pm 0.8$ & 0.002 \\
\hline Below 2/3rd RDA, n (\%) & $198(40)$ & $71(29.1)$ & $127(50.6)$ & $<0.001$ \\
\hline
\end{tabular}

Abbreviations: $\mu g$ micrograms; $m g$ milligrams; RAE Retinol Activity Equivalents; RDA Recommended Daily Allowance; SE standard error;

* Only including participants with completed dietary intake, and excluding those with Kcal outliers

$\mathbb{P}^{\mathbb{F}}$ For the analysis pertinent to the mean intake: Analysis of covariance (ANCOVA) was performed after adjusting for age and governorates. For chi-square anaIysis: No controlling for covariates was made

Bolded numbers are significant at $p<0.05$

\section{Abbreviations}

24-HR: $24 \mathrm{~h}$ recall; ANCOVA: Analysis of Covariance; El: Energy intake; EMR: Eastern Mediterranean Region; GBD: Global Burden of Disease; RAE: Retinol Activity Equivalents; RDA: Recommended Daily Allowance; SDGs: Sustainable Development Goals; SE: Standard error; SPSS: Statistical Package for Social Sciences; UK: United Kingdom; UNDP: United Nations Development Programme; USDA: United States Department of Agriculture; WHO: World Health Organization

\section{Acknowledgments}

The authors would like to thank Ms. Mandy Taktouk for her help in editing the manuscript.

\section{Authors' contributions}

Conceptualization, FN and LN; Methodology, NH and AMS; Data collection: MCC; Data analysis, JA; Writing—original draft preparation, FN and LN; Writing-review and editing, FN, LN, and MCC. All authors read and approved the final manuscript.

\section{Funding}

This research was supported by a grant from the Lebanese National Council for Scientific Research (LNCSR). The funder had no role in the study design, data collection, analysis, interpretation of data, and writing the manuscript.

\section{Availability of data and materials}

The datasets used and/or analyzed during the current study are available from the corresponding author on reasonable request.

\section{Ethics approval and consent to participate}

The design and conduct of the survey were approved by the Institutional Review Board of the American University of Beirut, and informed consents from adults/parents and informed assents from children and adolescents were obtained prior to enrollment in the study.

\section{Consent for publication}

Not applicable.

\section{Competing interests}

The authors declare that they have no competing interests.

\section{Author details}

'Department of Nutrition and Food Science, Faculty of Agricultural and Food Sciences, American University of Beirut, Beirut, Lebanon. ${ }^{2}$ Department of Epidemiology and Population Health, Faculty of Health Sciences, American University of Beirut, Beirut, Lebanon.

Received: 10 January 2020 Accepted: 11 March 2020

Published online: 26 March 2020

\section{References}

1. Nutrition International. Gender equality and nutrition. 2019. https://www. nutritionintl.org/about/gender-equality-nutrition/. Accessed 18 Nov 2019.

2. Torgrimson BN, Minson CT. Sex and gender: what is the difference? J Appl Physiol. 2005;99:785-7.

3. Pardue M-L, Wizemann TM. Exploring the biological contributions to human health: does sex matter?: National Academies Press. Institute of Medicine; 2001.

4. Bennett E, Peters SA, Woodward M. Sex differences in macronutrient intake and adherence to dietary recommendations: findings from the UK biobank. BMJ Open. 2018:8(4):e020017.

5. Abassi MM, Sassi S, El Ati J, Gharbia HB, Delpeuch F, Traissac P. Gender inequalities in diet quality and their socioeconomic patterning in a nutrition transition context in the Middle East and North Africa: a cross-sectional study in Tunisia. Nutr J. 2019;18(1):18.

6. Li R, Serdula M, Bland S, Mokdad A, Bowman B, Nelson D. Trends in fruit and vegetable consumption among adults in 16 US states: behavioral risk factor surveillance system, 1990-1996. Am J Public Health. 2000;90(5):777. 
7. Imamura F, Micha R, Khatibzadeh S, Fahimi S, Shi P, Powles J, et al. Dietary quality among men and women in 187 countries in 1990 and 2010: a systematic assessment. Lancet Glob Health. 2015;3(3):e132-e42.

8. Sudo N, Sekiyama M, Watanabe C, Mozammel Haque Bokul A, Ohtsuka R. Gender differences in food and energy intake among adult villagers in northwestern Bangladesh: a food frequency questionnaire survey. Int J Food Sci Nutr. 2004;55(6):499-509.

9. Arganini C, Saba A, Comitato R, Virgili F, Turrini A. Gender differences in food choice and dietary intake in modern western societies. Public healthsocial and behavioral health. London, UK: IntechOpen; 2012.

10. NCD Risk Factor Collaboration. Trends in adult body-mass index in 200 countries from 1975 to 2014: a pooled analysis of 1698 population-based measurement studies with 19.2 million participants. Lancet. 2016;387(10026): 1377-96.

11. Kanter R, Caballero B. Global gender disparities in obesity: a review. Adv Nutr. 2012;3(4):491-8.

12. Hwalla N, Al Dhaheri AS, Radwan H, Alfawaz HA, Fouda MA, Al-Daghri NM, et al. The prevalence of micronutrient deficiencies and inadequacies in the Middle East and approaches to interventions. Nutrients. 2017;9(3):229.

13. Hammarström A, Johansson K, Annandale E, Ahlgren C, Aléx L, Christianson $M$, et al. Central gender theoretical concepts in health research: the state of the art. J Epidemiol Community Health. 2014;68(2):185-90.

14. Krieger N. Genders, sexes, and health: what are the connections - and why does it matter? Int J Epidemiol. 2003;32(4):652-7.

15. Hassan R. Women in Islam: Quranic ideals versus Muslim realities. Plan Parent Chall. 1995;2:5-9.

16. Marshall S, Stokes R. Tradition and the veil: female status in Algeria and Tunisia. J Mod Afr Stud. 1981;19:625-46.

17. United Nations Development Programme - Lebanon. Sustainable Development Goals. Goal 5: Gender equality. 2019. https://www.lb.undp. org/content/lebanon/en/home/sustainable-development-goals/goal-5gender-equality.html. Accessed 20 Nov 2019.

18. United Nations Development Programme. Human Development Indices and Indicators: 2018 Statistical Update. Briefing note for countries on the 2018 Statistical Update: Lebanon 2018. http://hdr.undp.org/sites/all/themes/ hdr theme/country-notes/LBN.pdf. Accessed 29 Nov 2019.

19. Shawn F, Glenn F. Trends in global gender inequality (forthcoming, social forces). Soc Forces. 2010;88(5):1941-68.

20. Chamieh MC, Moore HJ, Summerbell C, Tamim H, Sibai AM, Hwalla N. Diet, physical activity and socio-economic disparities of obesity in Lebanese adults: findings from a national study. BMC Public Health. 2015;15(1):279.

21. Nasreddine L, Naja F, Akl C, Chamieh M, Karam S, Sibai A-M, et al. Dietary, lifestyle and socio-economic correlates of overweight, obesity and central adiposity in Lebanese children and adolescents. Nutrients. 2014;6(3):1038-62.

22. Naja F, Nasreddine L, Itani L, Chamieh MC, Adra N, Sibai AM, et al. Dietary patterns and their association with obesity and sociodemographic factors in a national sample of Lebanese adults. Public Health Nutr. 2011;14(9):1570-8.

23. Naja F, Hwalla N, Itani L, Karam S, Sibai AM, Nasreddine L. A Western dietary pattern is associated with overweight and obesity in a national sample of Lebanese adolescents (13-19 years): a cross-sectional study. Br J Nutr. 2015; 114(11):1909-19.

24. Lebanese Republic Ministry of Social Affairs. Central Administration for Statistics and the United Nations Development Programme (UNDP): Living Conditions of Households - The National Survey of Household Living Conditions. Beirut: MOSA. 2004. http://www.databank.com.lb/docs/Living\%2 Oconditions\%202004\%20-\%20CAS.pdf. Accessed 20 Nov 2019.

25. United Nations Fund for Population Activities (UNFPA) and the Lebanese Republic Ministry of Social Affairs. Statistical Tables for the Population and Housing Survey (PHS, 1994-1996). Beirut: MOSA 1996.

26. Melki I, Beydoun H, Khogali M, Tamim H, Yunis K. Household crowding index: a correlate of socioeconomic status and inter-pregnancy spacing in an urban setting. J Epidemiol Community Health. 2004;58(6):476-80.

27. Riva M, Plusquellec $P$, Juster R-P, Laouan-Sidi EA, Abdous B, Lucas $M$, et al. Household crowding is associated with higher allostatic load among the Inuit. J Epidemiol Community Health. 2014:jech-2013-203270.

28. Moshfegh AJ, Borrud L, Perloff B, LaComb R. Improved method for the 24hour dietary recall for use in national surveys. FASEB J. 1999;13(4):A603.

29. Moshfegh AJ, Rhodes DG, Baer DJ, Murayi T, Clemens JC, Rumpler WV, et al. The US Department of Agriculture Automated Multiple-Pass Method reduces bias in the collection of energy intakes. Am J Clin Nutr. 2008;88(2): 324-32.
30. Centers for Disease Control and Prevention - National Center for Health Statistics. Measuring Guides for the Dietary Recall Interview. 2015. https:// www.cdc.gov/nchs/nhanes/measuring_guides_dri/measuringguides.htm. Accessed 12 Nov 2019.

31. Pellet P, Shadarevian S. Food composition tables for use in the Middle East. Beirut, Lebanon: American University of Beirut; 2013.

32. Hoaglin DC, Iglewicz B. Fine-tuning some resistant rules for outlier labeling. J Am Stat Assoc. 1987:82(400):1147-9.

33. Scientific Advisory Committee on Nutrition. Dietary reference values for energy. London: TSO; 2011. https://assets.publishing.service.gov.uk/ government/uploads/system/uploads/attachment_data/file/339317/SACN_ Dietary_Reference_Values_for_Energy.pdf. Accessed 21 Nov 2019.

34. Kiefer I, Rathmanner T, Kunze M. Eating and dieting differences in men and women. J Mens Health Gend. 2005;2(2):194-201.

35. Naja F, Hwalla N, Itani L, Baalbaki S, Sibai A, Nasreddine L. A novel Mediterranean diet index from Lebanon: comparison with Europe. Eur J Nutr. 2015;54(8):1229-43.

36. Nasreddine L, Hwalla N, Sibai A, Hamzé M, Parent-Massin D. Food consumption patterns in an adult urban population in Beirut, Lebanon. Public Health Nutr. 2006;9(2):194-203.

37. Fagerli RA, Wandel M. Gender differences in opinions and practices with regard to a" healthy diet". Appetite. 1999;32(2):171-90.

38. Nasreddine L, Ayoub JJ, Hachem F, Tabbara J, Sibai AM, Hwalla N, et al. Differences in dietary intakes among Lebanese adults over a decade: results from two National Surveys 1997-2008/2009. Nutrients. 2019;11(8):1738.

39. FAO/WHO. Interim summary of conclusions and dietary recommendations on total fat and fatty acids. Joint FAO/WHO Expert Consultation on Fats and Fatty Acids in Human Nutrition. 2010. http://www.who.int/nutrition/topics/ FFA_summary_rec_conclusion.pdf. Accessed 21 Nov 2019.

40. Public Health England. National Diet and Nutrition Survey: results from years 1-4 combined of the rolling programme (2008 and 2009 to 2011 and 2012). London, UK: Public Health England. 2014.

41. Nasreddine L, Shatila H, Itani L, Hwalla N, Jomaa L, Naja F. A traditional dietary pattern is associated with lower odds of overweight and obesity among preschool children in Lebanon: a cross-sectional study. Eur J Nutr. 2019;58(1):91-102.

42. Nasreddine L, Tamim H, Itani L, Nasrallah MP, Isma'eel H, Nakhoul NF, et al. A minimally processed dietary pattern is associated with lower odds of metabolic syndrome among Lebanese adults. Public Health Nutr. 2018;(1): 160-71.

43. Liebman M, Cameron B, Carson D, Brown D, Meyer S. Dietary fat reduction behaviors in college students: relationship to dieting status, gender and key psychosocial variables. Appetite. 2001;36(1):51-6.

44. Beardsworth A, Bryman A, Keil T, Goode J, Haslam C, Lancashire E. Women, men and food: the significance of gender for nutritional attitudes and choices. Br Food J. 2002;104(7):470-91.

45. Beer-Borst S, Hercberg S, Morabia A, Bernstein M, Galan P, Galasso R, et al. Dietary patterns in six European populations: results from EURALIM, a collaborative European data harmonization and information campaign. Eur Clin Nutr. 2000;54(3):253.

46. Fulkerson JA, French SA, Story M. Adolescents' attitudes about and consumption of low-fat foods: associations with sex and weight-control behaviors. J Am Diet Assoc. 2004;104(2):233-7.

47. Pollard J, Kirk SL, Cade JE. Factors affecting food choice in relation to fruit and vegetable intake: a review. Nutr Res Rev. 2002;15(2):373-87.

48. Prättälä R, Paalanen L, Grinberga D, Helasoja V, Kasmel A, Petkeviciene J. Gender differences in the consumption of meat, fruit and vegetables are similar in Finland and the Baltic countries. Eur J Pub Health. 2006;17(5):520-5.

49. Wardle J, Parmenter K, Waller J. Nutrition knowledge and food intake. Appetite. 2000;34(3):269-75.

50. Wardle J, Haase AM, Steptoe A, Nillapun M, Jonwutiwes K, Bellisie F. Gender differences in food choice: the contribution of health beliefs and dieting. Ann Behav Med. 2004;27(2):107-16.

51. American University of Beirut - Lebanese National Council for Scientific Research. The Food-Based Dietary Guideline Manual for Promoting Healthy Eating in the Lebanese Adult Population. 2013. https://www.aub.edu.lb/fafs/ nfsc/Pages/Outreach.aspx. Accessed 25 Nov 2019.

52. Food and Agriculture Organization of the United Nations. Food-based dietary guidelines. 2019. http://www.fao.org/nutrition/education/fooddietary-guidelines/home/en/. Accessed 2 December 2019. 
53. Afshin A, Sur PJ, Fay KA, Cornaby L, Ferrara G, Salama JS, et al. Health effects of dietary risks in 195 countries, 1990-2017: a systematic analysis for the global burden of disease study 2017. Lancet. 2019;393(10184):1958-72.

54. Wansink B, Cheney MM, Chan N. Exploring comfort food preferences across age and gender. Physiol Behav. 2003;79(4-5):739-47.

55. Katou Y, Mori T, Ikawa Y. Effect of age and gender on attitudes towards sweet foods among Japanese. Food Qual Prefer. 2005;16(2):171-9.

56. Sobal J. Men, meat, and marriage: models of masculinity. Food Foodways. 2005;13(1-2):135-58.

57. World Health Organization. Regional Committee for the Eastern Mediterranean. Regional Strategy on Nutrition 2010-2019. EM/RC57/4. 2010. http:/applications. emro.who.int/docs/EM_RC57_4_en.pdf. Accessed 25 Nov 2019

58. Al Khatib L, Obeid O, Sibai A-M, Batal M, Adra N, Hwalla N. Folate deficiency is associated with nutritional anaemia in Lebanese women of childbearing age. Public Health Nutr. 2006:9(7):921-7.

59. Bailey RL, West KP Jr, Black RE. The epidemiology of global micronutrient deficiencies. Ann Nutr Metab. 2015;66(Suppl. 2):22-33.

60. Fenech MF. Dietary reference values of individual micronutrients and nutriomes for genome damage prevention: current status and a road map to the future. Am J Clin Nutr. 2010:91(5):1438S-54S.

61. van Ommen B, Wopereis S. Next-generation biomarkers of health. Nextgeneration nutritional biomarkers to guide better health care. 84: Karger Publishers; 2016. p. 25-33.

62. Avis WR. Gender equality and women's empowerment in Lebanon. Knowledge, evidence and learning for development: University of Birmingham; 2017. https://reliefweb.int/sites/reliefweb.int/files/resources/1 75-Gender-Equality-and-Womens-Empowerment-in-Lebanon.pdf. Accessed 20 Feb 2020.

63. Hamieh CS, Usta J. The effects of socialization on gender discrimination and violence. Oxfam Research Reports. Oxfam GB; 2011. https://pdfs. semanticscholar.org/1f84/253103dcd5d0ca3bcae21abf03abf0908b10.pdf. Accessed 20 Feb 2020

64. Wang G-J, Volkow ND, Telang F, Jayne M, Ma J, Rao M, et al. Exposure to appetitive food stimuli markedly activates the human brain. Neuroimage. 2004;21(4):1790-7.

65. Jomaa L, Naja F, Cheaib R, Hwalla N. Household food insecurity is associated with a higher burden of obesity and risk of dietary inadequacies among mothers in Beirut, Lebanon. BMC Public Health. 2017;17(1):567.

66. PMdCC $P, A F d R B ~ V$. Meat nutritional composition and nutritive role in the human diet. Meat Sci. 2013;93(3):586-92.

67. Caswell JA, Yaktine AL, Council NR. Individual, household, and environmental factors affecting food choices and access. Supplemental Nutrition Assistance Program: Examining the Evidence to Define Benefit Adequacy: National Academies Press (US); 2013.

68. Livingstone M, Robson P. Measurement of dietary intake in children. P Nutr Soc. 2000:59(2):279-93.

69. Burrows TL, Martin RJ, Collins CE. A systematic review of the validity of dietary assessment methods in children when compared with the method of doubly labeled water. J Am Diet Assoc. 2010;110(10):1501-10.

70. Conway JM, Ingwersen LA, Moshfegh AJ. Accuracy of dietary recall using the USDA five-step multiple-pass method in men: an observational validation study. J Am Diet Assoc. 2004;104(4):595-603.

71. Conway JM, Ingwersen LA, Vinyard BT, Moshfegh AJ. Effectiveness of the US Department of Agriculture 5-step multiple-pass method in assessing food intake in obese and nonobese women. Am J Clin Nutr. 2003:77(5):1171-8.

72. United Nations Entity for Gender Equality and the Empowerment of Women. Women and sustainable development goals. 2016. https:// sustainabledevelopment.un.org/content/documents/2322UN\%20Women\%2 0Analysis\%20on\%20Women\%20and\%20SDGs.pdf. Accessed 2 Dec 2019.

\section{Publisher's Note}

Springer Nature remains neutral with regard to jurisdictional claims in published maps and institutional affiliations.

Ready to submit your research? Choose BMC and benefit from:

- fast, convenient online submission

- thorough peer review by experienced researchers in your field

- rapid publication on acceptance

- support for research data, including large and complex data types

- gold Open Access which fosters wider collaboration and increased citations

- maximum visibility for your research: over $100 \mathrm{M}$ website views per year

At $\mathrm{BMC}$, research is always in progress.

Learn more biomedcentral.com/submissions 